An International elournal of Prehospital Care Research, Education, clinical Practice, Policy and Service Delivery ISSN 1447-4999

Evidence Based Practice

\title{
OXYGEN FOR REDUCING NAUSEA AND VOMITING DURING EMERGENCY AMBULANCE TRANSPORTATION: A SYSTEMATIC REVIEW OF RANDOMISED CONTROLLED TRIALS
}

Article No. 990018

Erin Smith, BHealthInfoMgt - Research Assistant, Monash University Centre for Ambulance and Paramedic Studies

\begin{abstract}
Objective: To determine the effectiveness of oxygen in decreasing nausea and vomiting during emergency transport.

Design: Systematic review of randomised controlled trials investigating the use of oxygen for reducing nausea and vomiting during emergency ambulance transport.

Participants: Patients with nausea and vomiting during emergency ambulance transport.

Results: Systematic review of the literature identified one trial suitable for review. The randomised controlled trial investigated the use of oxygen to reduce nausea and vomiting during emergency transport of patients with minor trauma. The trial reported that supplemental oxygen reduced nausea scores by $50 \%$ and reduced vomiting (a mean of 4 vomiting episodes in the oxygen group compared to a mean of 19 vomiting episodes in the room air group). These results are very similar to previous literature on the use of oxygen for post operative nausea and vomiting. However, the author's choice of statistical analysis compromises the quality of the trial and the significance of the results.
\end{abstract}

Conclusions: Oxygen as an intervention is inexpensive and has been successful in the treatment of perioperative and postoperative nausea and vomiting. Oxygen may be a simple method of curbing uncomfortable and potentially dangerous vomiting in patients being transported by ambulance. The use of 100\% oxygen therapy in the prehospital environment for nausea, motion sickness and vomiting is worthy of further research.

\section{Introduction}

Nausea, motion sickness and vomiting increases the discomfort of patients with traumatic injuries and further distress uncomfortable and anxious patients transported by ambulance. Medical implications of ambulance patients vomiting include the risk of powerful muscular contractions associated with vomiting leading to further damage in specific cases of trauma. The act of vomiting may subsequently result in dehydration, regurgitation of stomach contents (leading to risk of respiratory obstruction, pulmonary inflammation and aspiration pneumonia), increases in intracranial pressure (risking further damage to patients with ocular 
injuries), added pressure on abdominal wounds, and impact on vagal stimulation causing changes in blood pressure and pulse rate.

Previous studies have shown a correlation between the use of supplemental oxygen in perioperative and postoperative environments and a decrease in nausea and vomiting. These results support the theory that oxygen has some antiemetic properties.1 Raising the concentration of perioperative inspired oxygen may reduce the incidence of nausea and vomiting by half after abdominal surgery.2 Greif and associates found that patients who received the standard 30\% concentration of oxygen during and after colorectal surgery were almost twice as likely to experience nausea than those receiving an $80 \%$ oxygen concentration.

A potential toxic effect of oxygen administration is pulmonary atelectasis.3 Pulmonary atelectasis is defined as an abnormal condition characterised by the collapse of the alveoli, preventing the respiratory exchange of carbon dioxide and oxygen, and resulting in increasing dyspnea.4 The literature shows that even a brief exposure to $100 \%$ oxygen can cause demonstrable atelectasis.3 However, despite the risk of atelectasis, the problem can be completely reversed with a single positive pressure breath, and has little effect on gas exchange or lung function.5 Recent literature has concluded that supplemental oxygen therapy in the perioperative period does not cause prolonged atelectasis or permanent lung function impairment.5 Following from these findings, we can assume that supplemental use of $100 \%$ oxygen in the prehospital environment will not result in pulmonary atelectasis.

Pulmonary inflammation is another potential toxic effect of $100 \%$ oxygen therapy. However, this has only been proven in cases where perioperative oxygen therapy was continued for longer than six hours, and therefore not applicable to patients receiving brief periods of $100 \%$ oxygen therapy while being transported to hospital. 100\% oxygen therapy should be used with caution in patients with pre existing chronic obstructive airway disease (COAD) 2001).6 When COAD patients inhale $100 \%$ oxygen the reduction in hypoxia reduces ventilation, potentially increasing the retention of carbon dioxide.7 The issue of inspired oxygen concentration remains controversial, and its antiemetic effect is worthy of further research.

\section{Methods}

\section{Inclusion criteria}

The literature search was designed to identify all randomised controlled trials (RCTs) and quasi-RCTs investigating the use of oxygen for reducing nausea and vomiting during emergency ambulance transport. Relevant trials were those where patients were assigned to receive oxygen or alternative protocols based on a randomised or quasi-randomised method. We included trials that compared oxygen to no oxygen, oxygen to room air, and oxygen to anti-emetic. Participants were eligible for inclusion if they required emergency ambulance transport for any reason, and had nausea and/or vomiting prior to transport, or had developed nausea and/or vomiting during transport.

\section{Identification of trials}

A comprehensive literature search was conducted identifying studies that appeared potentially relevant to the review. Abstracts for these studies were assessed, and relevant full text articles were retrieved. Reviewers used the full text of the relevant papers to identify those suitable for review. In all instances, differences of opinion were resolved by re-assessing the paper and communication between the three reviewers. The Cochrane Controlled Trials Register (The Cochrane Library, Issue 3, 2002), MEDLINE, using OVID (1966 - Jan Week 2, 2003), 
CINAHL (1982 - Jan Week 5, 2003), The Australasian Medical Index (AMI) (1968 current) and the web based search engines Google and Yahoo were searched using each of the text terms "nausea", "vomit", “emesis", “emetic", “motion sickness" and "oxygen". Reference lists of all relevant publications were searched, conference proceedings and contents of key Pre-Hospital journals not indexed on electronic databases were hand searched when necessary, and no language restrictions applied.

\section{Outcome measures}

The principal outcome measures were decrease in nausea sensation, motion sickness sensation and vomiting. The anti-emetic efficacy of oxygen was assessed using incidence of the following: reported nausea sensation, reported motion sickness or any vomiting episode (including retching). Additional outcome measures assessed included patient satisfaction with ambulance transport, and adverse reactions to oxygen therapy.

\section{Data extraction and quality assessment}

Reviewers extracted data independently using a form designed for the review (see appendix one). Data was extracted on type of participants, interventions and outcome measures, methods of randomisation, allocation concealment, blinding, losses to follow up and results (see appendix one). Quality was assessed using an adaptation of the scale outlined by Schulz et al.8 assigning $\mathrm{C}$ to poorest quality and $\mathrm{A}$ to best quality.

Adequacy of randomisation process

A. Adequate sequence generation is reported using random number tables, computer random number generator, coin tossing, or shuffling.

B. Did not specify one of the adequate reported methods in (A) but mentioned a randomisation method.

C. Other methods of allocation that appear to be unbiased.

Adequacy of the allocation concealment process

A. Adequate measures to conceal allocations such as central randomisation, serially numbered, opaque, sealed envelopes; or other description that contains convincing elements of concealment.

B. Unclearly concealed trials in which the author either did not report an allocation concealment approach at all, or reported an approach that did not fall into one of the categories in (A).

C. Inadequately concealed trials in which the method of allocation is not concealed, such as alteration methods or use of case numbers.

Potential for selection bias after allocation

A. Trials where an intention to treat analysis is possible and few losses to follow up are noted.

B. Trials which reported exclusions (as listed in A, but exclusions were less than $10 \%$ )

C. No reporting on exclusions or exclusions greater than $19 \%$, or wide differences in exclusions between groups.

Level of masking

A. Double or triple blind.

B. Single blind.

C. Non blind. 


\section{$\underline{\text { Results }}$}

The literature search identified one randomised controlled trial (RCT) suitable for inclusion in the review. The trial with 100 patients by Kober et al (2002) was conducted between January - April 2000 and investigated the use of oxygen in reducing nausea and vomiting during emergency transport of patients older than 60 years with minor trauma. Patient inclusion criteria were $>60$ years of age with minor trauma sufficient to require ambulance transport to hospital. Only patients who could be transported safely after simple bandaging and immobilization were included in the study. Patients with acute or chronic respiratory disease were excluded.

The trial by Kober et al. found that supplemental oxygen reduced nausea scores by $50 \%$ and reduced vomiting episodes (A mean of 4 episodes in oxygen group compared to a mean of 19 episodes in room air group). The authors reported a significant improvement in overall satisfaction within the oxygen therapy group. As patients cannot consciously distinguish between different concentrations of inspired oxygen, the authors concluded that the findings were due to the reduction of nausea and vomiting in the oxygen group.5

Using the scale defined by Schulz the quality of the trial was of a high standard, scoring A's. Patients were allocated to either treatment or control group using computer generated codes that were maintained in sequentially numbered opaque envelopes.8 The intervention was $100 \%$ oxygen at $10 \mathrm{~L} / \mathrm{min}$ through a face mask, and the comparison was room air through a face mask. Opaque envelopes were also used for allocation concealment. The paramedic who treated the patient in the back of the ambulance opened the randomisation envelope after each patient was secured in the back of the ambulance. The oxygen or room air was contained in 2 cylinders which were located in the back of the ambulance with the unblinded paramedic. On arrival at hospital, a paramedic who was blind to the intervention interviewed the patient. Patients cannot distinguish between different concentrations of inspired oxygen, so we can assume they were blinded to the intervention. Losses to follow up and intention to treat were not addressed.

The results from the two groups were compared with CHI SQUARE or unpaired 2-tailed $t$ tests, and presented as means \pm standard deviations. The statistical analysis of this trial found significant differences between the oxygen and room air groups, with the mean nausea rating significantly lower in the oxygen group. The authors have assessed patient nausea scores on a visual analog scale (VAS), however, they have not provided us with the possible range of values, or indicated whether a VAS scale was used in the measurement of overall patient satisfaction. We can assume that the VAS scale must start at zero as patients cannot report negative nausea. The mean value for the oxygen group is given as 22, indicating that this is representative of the middle of the population. The standard deviation of 29 suggests that the population is highly skewed and therefore not normally distributed. This raises concern regarding the choice of statistical analysis as CHI SQUARE assumes normal distribution of a population.

Both intervention and comparison groups had similar baseline characteristics. They were comparable for gender distribution, age and weight. Both groups had comparable initial systolic and diastolic blood pressure, heart rate, oral temperature and anxiety level. Transport duration for both groups did not differ significantly. The results of the trial are presented in Table 1. 


\begin{tabular}{|l|l|}
\hline Oxygen group & Room air group \\
\hline $\begin{array}{l}\text { Higher oxygen saturation } \\
(99 \% \pm 1 \% ; P<.001)\end{array}$ & $\begin{array}{l}\text { Lower oxygen saturation } \\
(96 \% \pm 2 \%: P<.001)\end{array}$ \\
\hline $\begin{array}{l}\text { Greater pain and nausea scores before } \\
\text { randomisation }\end{array}$ & $\begin{array}{l}\text { Lower pain and nausea scores before } \\
\text { randomisation }\end{array}$ \\
\hline $\begin{array}{l}\text { Less nausea after oxygen } \\
(22 \pm 29 ; P<.001)\end{array}$ & $\begin{array}{l}\text { More nausea after room air } \\
(54 \pm 38 ; P<.001)\end{array}$ \\
\hline Less vomiting $(4$ episodes) & More vomiting $(19$ episodes $)$ \\
\hline $\begin{array}{l}\text { Lower heart rates } \\
(86 \pm 12 ; P<.001)\end{array}$ & $\begin{array}{l}\text { Higher heart rates } \\
(94 \pm 13 ; P<.001)\end{array}$ \\
\hline $\begin{array}{l}\text { Greater overall satisfaction } \\
(54 \pm 33 ; P<.001)\end{array}$ & $\begin{array}{l}\text { Lower overall satisfaction } \\
(33 \pm 23 ; P<.001)\end{array}$ \\
\hline
\end{tabular}

Table 1: Results from Kober et al (2002)

\section{Discussion}

Our literature search identified numerous papers addressing the use of oxygen to reduce nausea and vomiting in the hospital post operative setting, but identified only one PreHospital based RCT that met the inclusion criteria for the review. The overall quality of the trial was good scoring A's on the scale outlined by Schulz et al,8 assigning $C$ to poorest quality and A to best quality. However, the method of statistical analysis is questionable. This did not make the data invalid, but suggests a need to reassess the results using nonparametric statistical methods. The results from such a reanalysis may identify different responses to oxygen therapy for nausea and vomiting than the significant results reported in this trial.

The authors have failed to account for confounding variables which may be responsible for the differences seen between the two groups. The authors state that supine position and patient inability to see outside of the ambulance are contributing factors to motion sickness, however they do not identify any differences in posture of patients enrolled in this study. We are given no information regarding the speeds at which the ambulances were travelling, whether all patients were taken the same, or a similar route to hospital, or if some patients experienced travel at higher speeds and with more bends, stops and starts. All of these factors have the potential to induce nausea and vomiting. The authors address specific extraneous factors, including that patients in the oxygen group were slightly taller, had significantly more pain before transport, and a higher incidence of pre existing nausea. However the authors argue that there is no proven association between height and motion sickness, or pain and nausea.

An important question not raised by the authors was that of analgesia. The patients in the study were reporting initial mean pain scores of 50-65 mm on the VAS scale. This is a level of pain that would usually warrant some form of analgesic intervention. The authors do not state whether analgesics were administered, and therefore we have no way to determine if analgesic administration influenced the incidence of nausea and vomiting in either of the study groups.

Bias was not addressed in the trial, however selection bias is possible due to the trial only including patients $>60$ years of age with minor trauma. Limitations were not addressed, 
however it would be difficult to generalise this trial to other patient populations due to the limits placed on age and severity of trauma. The trial does not state if the unblinded paramedic stayed in the rear of the ambulance for the duration of the transport, or if the blinded paramedic was a driver who only accessed the patient on arrival at hospital. The authors do not elucidate whether all patients were driven to hospital by the same paramedic team, and we cannot eliminate the possibility that the blind paramedic had access to controls in the front of the ambulance which may have alerted them as to which treatment the patient was receiving.

Finally, the authors fail to acknowledge the potential contamination of $100 \%$ oxygen with room air when using the standard ventilation masks. 100\% oxygen can only be achieved with the use of a closed circuit oxygen mask. The authors do not provide details as to the type of oxygen masks used, and therefore conclusions cannot be inferred as to the purity of the $100 \%$ oxygen administered.

\section{Limitations}

In common with any Pre-Hospital literature review, several issues are highlighted. Firstly, the lack of published Pre-Hospital RCTs. Systematic review of the literature has identified 60 Pre-Hospital RCTs, 40 of which were cardiac related. Secondly, the difficulty of identifying relevant Pre-Hospital literature using electronic database searches. Problems with indexing and a lack of specialist MeSH terms for the Pre-Hospital field are contributing problems. This demonstrates the need to hand search applicable journals, search other database systems such as the Australian Medical Index (AMI) and various web based search engines, and contact authors and researchers known to work in the field when attempting any systematic review in the Pre-Hospital environment.

\section{Implications for practice}

Oxygen as in intervention is inexpensive and has been successful in the treatment of perioperative and postoperative nausea and vomiting. Oxygen may be a simple method of curbing uncomfortable and potentially dangerous vomiting in patients being transported by ambulance, however, there is currently a lack of evidence to implement oxygen as an antiemetic in emergency ambulance transport. The use of oxygen therapy in the Pre-Hospital environment for nausea, motion sickness and vomiting is worthy of further research.

Without statistical results from other similar RCTs to pool and examine, we conclude that there is not enough evidence to implement the use of oxygen as an anti-emetic in emergency ambulance transport at this time. 


\section{References}

1. Overdyk FJ, Roy RC. If nitrous oxide induces emesis, maybe $100 \%$ oxygen is an antiemetic (letter). Anesth Analg 1997;84:228-236

2. Greif R, Laciny S, Rapf B, Hickle RS, Sessler DI. Supplemental oxygen reduces the incidence of postoperative nausea and vomiting. Anaesthesiology. 1999;91:12461252.

3. Hedenstierna G. Atelectasis and its prevention during anaesthesia. European Journal Anaesthesiology. 1998;15:387-390.

4. Anderson KN (ed) et al. Mosby's Medical, Nursing and Allied Health Dictionary. Fifth edition. Mosby-Year Book, Inc. 1998.

5. Kober A, Fleischackl R, Scheck T, Lieba F, Strasser H, Friedmann A, Sessler DI. A Randomized Controlled Trial of Oxygen for Reducing Nausea and Vomiting During Emergency Transport of Patients Older than 60 Years With Minor Trauma. Mayo Clinic Proceedings. 2002;77(1); 35-38.

6. Bach PB, Brown C, Gelfand SE, et al. Management of acute exacerbations of chronic obstructive pulmonary disease: a summary and appraisal of published evidence. Annals Internal Medicine. 2001;134: 600-620.

7. Rees PJ, Dudley F. Oxygen therapy in chronic lung disease. BMJ.1998;317: 871-874

8. Schulz K, Chalmers I, Hayes R, Altman D. Empirical evidence of bias - Dimensions of Methodological quality associated with estimates of treatment effects in controlled trials. JAMA. 1995;273(5):408-412

\section{Additional Reading}

1. Akca O, Podolsky A, Eisenhuber E, et al. Comparable postoperative pulmonary atelectasis in patients given $30 \%$ or $80 \%$ oxygen during and for 2 hours after colon resection. Anaesthesiology. 1999;91:991-998.

2. Joyce CJ, Baker AB. Effects of inspired gas composition during anaesthesia for abdominal hysterectomy on postoperative lung volumes. British Journal Anaesthesia. 1995;75:417-421.

3. Magnusson L, Zemgulis V, Tenling A, et al. Use of a vital capacity maneuver to prevent atelectasis after cardiopulmonary bypass: an experimental study. Anaesthesiology. 1998;88:134-14.

4. Rothen HU, Sporre B, Engberg G, Wegenius G, Hedenstierna G. Reexpansion of atelectasis during general anaesthesia may have a prolonged effect. Acta Anaesthsiol Scand. 1995;39:118-125. 


\section{Appendix One}

\section{SAMPLE DATA EXTRACTION FORM}

Reviewer Name

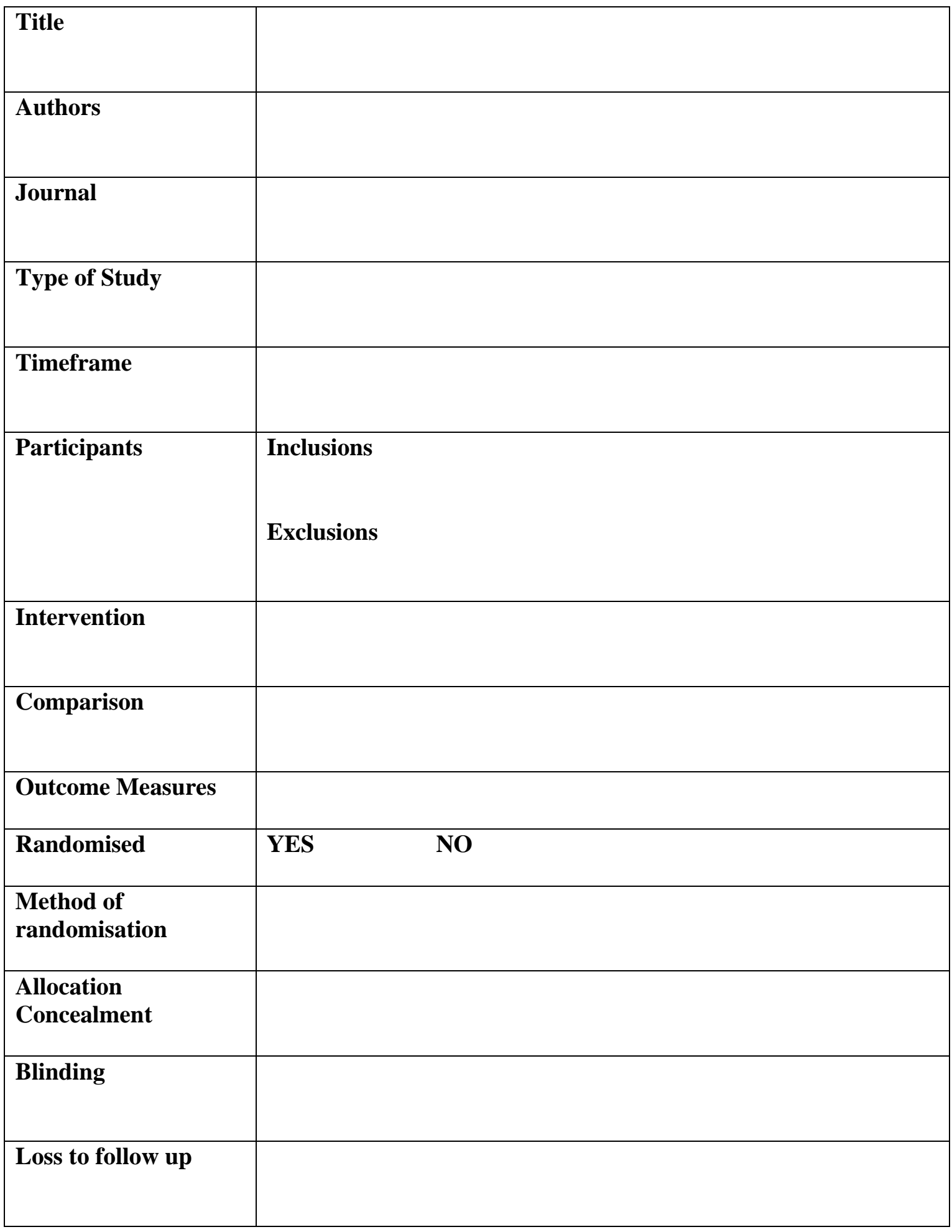




\begin{tabular}{|l|l|}
\hline Intention to treat & \\
\hline $\begin{array}{l}\text { Similar baseline } \\
\text { characteristics }\end{array}$ & \\
\hline Bias & \\
\hline Limitations & \\
\hline Results & \\
\hline
\end{tabular}

\section{Quality Assessment}

Quality was assessed using an adaptation of the scale outlined in Schulz (Schulz 1995), assigning $\mathrm{C}$ to poorest quality and $\mathrm{A}$ to best quality.

\section{Adequacy of randomisation process}

A. Adequate sequence generation is reported using random number tables, computer random number generator, coin tossing, or shuffling.

B. Did not specify one of the adequate reported methods in (A) but mentioned a randomisation method.

C. Other methods of allocation that appear to be unbiased.

\section{Adequacy of the allocation concealment process}

A. Adequate measures to conceal allocations such as central randomisation, serially numbered, opaque, sealed envelopes; or other description that contains convincing elements of concealment.

B. Unclearly concealed trials in which the author either did not report an allocation concealment approach at all, or reported an approach that did not fall into one of the categories in $(\mathrm{A})$.

C. Inadequately concealed trials in which the method of allocation is not concealed, such as alteration methods or use of case numbers. 


\section{Potential for selection bias after allocation}

A. Trials where an intention to treat analysis is possible and few losses to follow up are noted.

B. Trials which reported exclusions (as listed in A, but exclusions were less than 10\%)

C. No reporting on exclusions or exclusions greater than $19 \%$, or wide differences in exclusions between groups.

\section{Level of masking}

A. Double or triple blind.

B. Single blind.

C. Non blind.

\section{Overall Quality assessment}

A B C

\section{Notes}

\section{Acknowledgments:}

The author would like to acknowledge the contributions of co-reviewers Amee Cooper and Andrea Wyatt. 